PROCEEDINGS OF THE

AMERICAN MATHEMATICAL SOCIETY

Volume 124, Number 12, December 1996, Pages 3613-3618

S 0002-9939(96)03644-1

\title{
SOME REMARKS ON BEILINSON ADELES
}

\author{
AMNON YEKUTIELI
}

(Communicated by Eric M. Friedlander)

\begin{abstract}
Let $X$ be a scheme of finite type over a field $k$. Denote by $\mathcal{A}_{X}$ the sheaf of Beilinson adeles with values in the algebraic De Rham complex $\Omega_{X / k}$. Then $\Omega_{X / k}^{*} \rightarrow \mathcal{A}_{X}^{*}$ is a flasque resolution. So if $X$ is smooth, $\mathcal{A}_{X}$ calculates De Rham cohomology. In this note we rewrite the proof of Deligne-Illusie for the degeneration of the Hodge spectral sequence in terms of adeles. We also give a counterexample to show that the filtration $\mathcal{A}_{\dot{X}}^{\cdot \geq q}$ does not induce Hodge decomposition.
\end{abstract}

\section{INTRODUCTION}

In this note we consider two aspects of Beilinson adeles on schemes.

Let $X$ be a scheme of finite type over a field $k$. Given a quasi-coherent sheaf $\mathcal{M}$ let $\mathbb{A}_{\text {red }}^{q}(\mathcal{M})$ be the sheaf of reduced Beilinson adeles of degree $q$ (see [Be], [Hr], $[\mathrm{HY} 1])$. It is known that $\underline{\mathbb{A}}_{\text {red }}^{q}(\mathcal{M}) \cong \mathbb{\mathbb { A }}_{\text {red }}^{q}\left(\mathcal{O}_{X}\right) \otimes_{\mathcal{O}_{X}} \mathcal{M}$. For any open set $U \subset X$

$$
\Gamma\left(U, \mathbb{A}_{\mathrm{red}}^{q}(\mathcal{M})\right) \subset \prod_{\xi \in S(U)_{q}^{\mathrm{red}}} \mathcal{M}_{\xi}
$$

where $S(U)_{q}^{\text {red }}$ is the set of reduced chains of points in $U$ of length $q$, and $\mathcal{M}_{\xi}$ is the Beilinson completion of $\mathcal{M}$ along the chain $\xi$ (cf. [Ye1]). For $q=0$ and $\mathcal{M}$ coherent one has $\mathcal{M}_{(x)}=\widehat{\mathcal{M}}_{x}$, the $\mathfrak{m}_{x}$-adic completion, and (0.1) is an equality.

Let $\Omega_{X / k}$ be the De Rham complex on $X$, relative to $k$. As shown in [HY1], setting $\mathcal{A}_{X}^{p, q}:=\underline{\mathbb{A}}_{\mathrm{red}}^{q}\left(\Omega_{X / k}^{p}\right)$ and $\mathcal{A}_{X}^{i}:=\bigoplus_{p+q=i} \mathcal{A}_{X}^{p, q}$ we get a differential graded algebra (DGA) which is quasi-isomorphic to $\Omega_{X / k}$ and is flasque. Thus $\mathrm{H}^{\cdot}\left(X, \Omega_{X / k}^{\cdot}\right)=$ $\mathrm{H}^{\cdot} \Gamma\left(X, \mathcal{A}_{X}\right)$. In particular if $X$ is smooth, we get the De Rham cohomology $\mathrm{H}_{\mathrm{DR}}(X / k)$.

More generally, let $\mathfrak{X}$ be a formal scheme, of formally finite type (f.f.t.) over $k$ (see [Ye2]). Then applying the adelic construction to the complete De Rham complex $\widehat{\Omega}_{\mathfrak{X} / k}$ we get a DGA $\mathcal{A}_{\mathfrak{X}}$. If $X \subset \mathfrak{X}$ is a smooth formal embedding (op. cit.) and char $k=0$, then $\mathrm{H}^{\cdot} \Gamma\left(X, \mathcal{A}_{\mathfrak{X}}\right)=\mathrm{H}_{\mathrm{DR}}(X / k)$.

There is an analogy between the sheaf $\mathcal{A}_{X}^{p, q}$ on a smooth $n$-dimensional variety $X$ and the sheaf of smooth $(p, q)$-forms on a complex manifold. The coboundary operator $\mathrm{D}$ of $\mathcal{A}_{X}$ is defined as a sum $\mathrm{D}:=\mathrm{D}^{\prime}+\mathrm{D}^{\prime \prime}$, and $\mathrm{D}^{\prime \prime}: \mathcal{A}_{X}^{p, q} \rightarrow \mathcal{A}_{X}^{p, q+1}$ plays

Received by the editors May 24, 1995.

1991 Mathematics Subject Classification. Primary 14F40; Secondary 14C30, 13J10.

This research was partially supported by an Allon Fellowship. The author is an incumbent of the Anna and Maurice Boukstein Career Development Chair.

(C)1996 American Mathematical Society 
the role of the anti-holomorphic derivative. The map $\int_{X}=\sum_{\xi} \operatorname{Res}_{\xi}: \Gamma\left(X, \mathcal{A}_{X}^{2 n}\right) \rightarrow$ $k$ is the counterpart of the integral (Res $\xi$ is the Parshin-Lomadze residue along the maximal chain $\xi$ in $X$, see [Ye1]). This analogy to the complex manifold picture is quite solid; for example, in [HY2] there is an algebraic proof of the Bott residue formula, which in some parts is just a translation of the original proof of Bott to the setting of adeles.

The main purpose of this note is to examine the potential applicability of adeles for the study of algebraic De Rham cohomology. In $\S 1$ the construction of Deligne and Illusie [DI] is rewritten in terms of adeles. In $\S 2$ we consider a possibility to relate adeles to Hodge theory, and show by example its failure.

\section{LifTing MODUlO $p^{2}$}

We interpret, in terms of adeles, the result of Deligne and Illusie on the decomposition of the De Rham complex in characteristic $p$. In this section we shall follow closely the ideas and notation of $[\mathrm{DI}]$.

Let $k$ be a perfect field of characteristic $p$. Write $\tilde{k}:=W_{2}(k)$. Let $F_{k}:$ Spec $k \rightarrow$ Spec $k$ be the Frobenius morphism, i.e. $F_{k}^{*}(a)=a^{p}$ for $a \in k$. By pullback along $F_{k}$ we get a scheme $X^{\prime}:=X \times_{k} k$ and a finite, bijective $k$-morphism $F=F_{X / k}$ : $X \rightarrow X^{\prime}$.

Assume we are given some lifting $\tilde{X}$ of $X$ to $\tilde{k}$. By this we mean a smooth scheme $\tilde{X}$ over $\tilde{k}$ s.t. $X \cong \tilde{X} \times_{\tilde{k}} k$. Using the Frobenius $F_{\tilde{k}}$ we also define a scheme $\tilde{X}^{\prime}$, and a $\tilde{k}$-morphism $F_{\tilde{X}}: \tilde{X} \rightarrow \tilde{X}^{\prime}$. For any point $x \in X$ the relative Frobenius homomorphism $F_{x}^{*}: \mathcal{O}_{X^{\prime}, F(x)} \rightarrow \mathcal{O}_{X, x}$ can be lifted to a $\tilde{k}$-algebra homomorphism $\tilde{F}_{x}^{*}: \mathcal{O}_{\tilde{X}^{\prime}, F(x)} \rightarrow \mathcal{O}_{\tilde{X}, x}$ (cf. [DI]). In view of (0.1), the collection $\left\{\tilde{F}_{x}^{*}\right\}_{x \in X}$ induces a homomorphism of sheaves of DG $\tilde{k}$-algebras

$$
\tilde{F}^{*}: \underline{\mathbb{A}}_{\mathrm{red}}^{0}\left(\Omega_{\tilde{X}^{\prime} / \tilde{k}}^{\cdot}\right) \rightarrow F_{*} \underline{\mathbb{A}}_{\mathrm{red}}^{0}\left(\Omega_{\tilde{X} / \tilde{k}}^{\cdot}\right) .
$$

Lemma 1.1. The liftings $\left\{\tilde{F}_{x}^{*}\right\}_{x \in X}$ determine $\mathcal{O}_{X^{\prime}}$-linear homomorphisms

$$
\begin{gathered}
f: \Omega_{X^{\prime} / k}^{1} \rightarrow F_{*} \mathcal{A}_{X}^{1,0}, \\
h: \Omega_{X^{\prime} / k}^{1} \rightarrow F_{*} \mathcal{A}_{X}^{0,1}
\end{gathered}
$$

such that

$$
\mathrm{D}(f+h)=0 .
$$

Proof. Let $\mathbf{p}: \Omega_{X / k}^{\cdot} \stackrel{\simeq}{\rightarrow} p \Omega_{\tilde{X} / \tilde{k}}^{\cdot}$ be multiplication by $p$. This extends to an $\underline{\mathbb{A}}_{\text {red }}^{0}\left(\mathcal{O}_{X}\right)$ linear isomorphism

$$
\mathbf{p}: \mathcal{A}_{X}^{\cdot, 0}=\underline{\mathbb{A}}_{\mathrm{red}}^{0}\left(\Omega_{X / k}^{\cdot}\right) \stackrel{\simeq}{\rightarrow} p \underline{\mathbb{A}}_{\mathrm{red}}^{0}\left(\Omega_{\tilde{X} / \tilde{k}}^{\cdot}\right)
$$

Just as in [DI] we get a homomorphism $f$ making the diagram

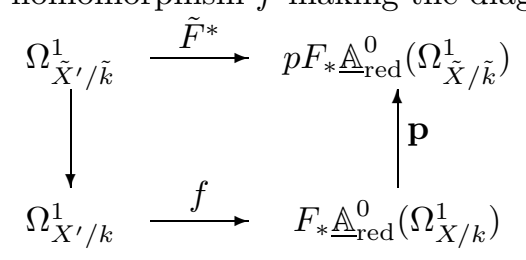

commutative. 
Next, for any chain of points $\left(x_{0}, x_{1}\right)$ in $X$ and a local section $a \in \mathcal{O}_{\tilde{X}^{\prime}}$ we have

$$
\mathrm{D}^{\prime \prime} \tilde{F}^{*}(a)=\tilde{F}_{x_{0}}^{*}(a)-\tilde{F}_{x_{1}}^{*}(a) \in p \mathcal{O}_{\tilde{X},\left(x_{0}, x_{1}\right)} .
$$

Therefore

$$
\mathrm{D}^{\prime \prime} \tilde{F}^{*}: \mathcal{O}_{\tilde{X}^{\prime}} \rightarrow p F_{*} \underline{\mathbb{A}}_{\text {red }}^{1}\left(\mathcal{O}_{\tilde{X}}\right)
$$

is a derivation which kills $p \mathcal{O}_{\tilde{X}^{\prime}}$, and we get an $\mathcal{O}_{X^{\prime}}$-linear homomorphism $h$ s.t. the diagram

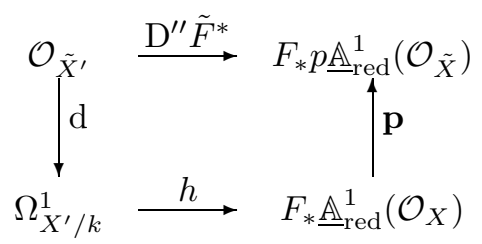

commutes.

Reinterpreting the calculations of [DI] in terms of adeles we see that the following hold: for each point $x_{0} \in X, \mathrm{D}^{\prime} f=0$ in $\Omega_{X / k,\left(x_{0}\right)}^{1}$; for each chain $\left(x_{0}, x_{1}, x_{2}\right)$ in $X$, $\mathrm{D}^{\prime \prime} h=0$ in $\mathcal{O}_{X,\left(x_{0}, x_{1}, x_{2}\right)}$; lastly, for each chain $\left(x_{0}, x_{1}\right), \mathrm{D}^{\prime \prime} f=-\mathrm{D}^{\prime} h$ in $\Omega_{X / k,\left(x_{0}, x_{1}\right)}^{1}$. This implies that on the level of sheaves $\mathrm{D}(f+h)=0$.

Proposition 1.2. The liftings $\left\{\tilde{F}_{x}^{*}\right\}_{x \in X}$ determine an $\mathcal{O}_{X^{\prime}}$-linear homomorphism of complexes

$$
\psi_{\tilde{X}}: \bigoplus_{i=0}^{n} \mathbb{A}_{\mathrm{red}}^{\cdot}\left(\Omega_{X^{\prime} / k}^{i}\right)[-i] \rightarrow F_{*} \mathcal{A}_{X}
$$

making the diagram

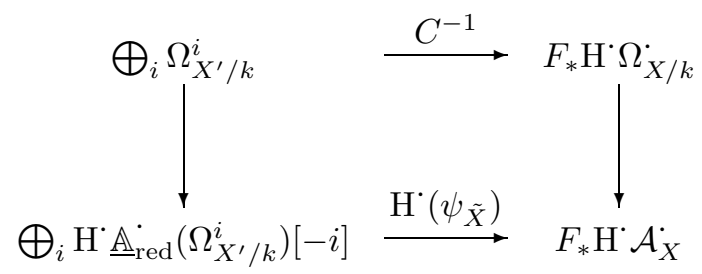

commute. Here $C^{-1}$ is the Cartier operation, and the vertical arrows are the canonical isomorphisms. Therefore $\psi_{\tilde{X}}$ is a quasi-isomorphism.

Proof. Since

$$
\underline{\mathbb{A}}_{\mathrm{red}}^{j}\left(\Omega_{X^{\prime} / k}^{i}\right) \cong \underline{\mathbb{A}}_{\mathrm{red}}^{j}\left(\mathcal{O}_{X^{\prime}}\right) \otimes_{\mathcal{O}_{X^{\prime}}} \Omega_{X^{\prime} / k}^{i}
$$

and since $F^{*}: \underline{\mathbb{A}}_{\text {red }}^{\cdot}\left(\mathcal{O}_{X^{\prime}}\right) \rightarrow F_{*} \underline{\mathbb{A}}_{\text {red }}^{\cdot}\left(\mathcal{O}_{X}\right)$ commutes with $\mathrm{D}^{\prime \prime}$ and is killed by $\mathrm{D}^{\prime}$ (i.e. $\mathrm{D}^{\prime} F^{*}=0$ ), it suffices to define $\mathcal{O}_{X^{\prime}}$-linear homomorphisms $\psi_{\tilde{X}}^{i}: \Omega_{X^{\prime} / k}^{i} \rightarrow F_{*} \mathcal{A}_{X}^{i}$ s.t. $\mathrm{D} \psi_{\tilde{X}}^{i}=0$. Define $\psi_{\tilde{X}}^{0}:=F^{*}$, and $\psi_{\tilde{X}}^{1}:=f+h$ as in Lemma 1.1. For $1 \leq i \leq n$ let $\mathbf{a}: \Omega_{X^{\prime} / k}^{i} \rightarrow\left(\Omega_{X^{\prime} / k}^{1}\right)^{\otimes i}$ be the anti-symmetrizing operator (this makes sense 
since $n<p$; cf. [DI]), and define $\psi_{\tilde{X}}^{i}$ by

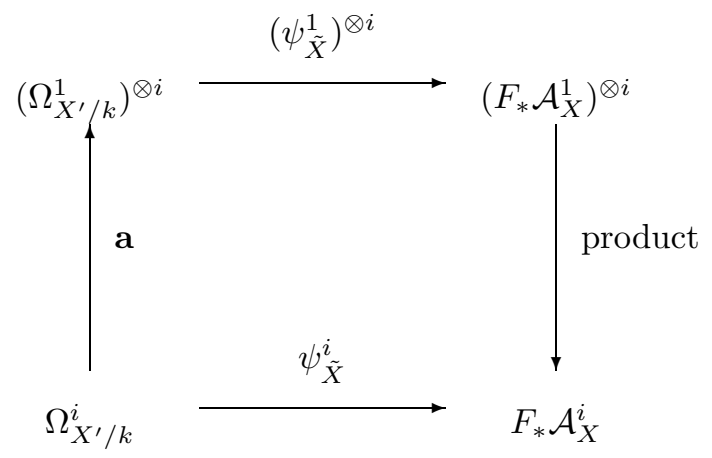

Let $a \in \mathcal{O}_{\tilde{X}}$ be a local section, with corresponding pullback $a \otimes 1 \in \mathcal{O}_{\tilde{X}^{\prime}}$, and with image $a_{0} \in \mathcal{O}_{X}$. Then according to the calculations in [DI], we have $\tilde{F}^{*}(a \otimes 1)=$ $a^{p}+\mathbf{p} u$ for some local section $u \in \mathbb{A}_{\text {red }}^{0}\left(\mathcal{O}_{X}\right)$. Therefore $f\left(\mathrm{~d} a_{0} \otimes 1\right)=a_{0}^{p-1} \mathrm{~d} a_{0}+\mathrm{D}^{\prime} u$ and $h\left(\mathrm{~d} a_{0} \otimes 1\right)=\mathrm{D}^{\prime \prime} u$, so $\psi_{\tilde{X}}\left(\mathrm{~d} a_{0} \otimes 1\right)=a_{0}^{p-1} \mathrm{~d} a_{0}+\mathrm{D} u$. This means that

$$
\mathrm{H}^{1}\left(\psi_{\tilde{X}}\right)=C^{-1}: \Omega_{X^{\prime} / k}^{1} \stackrel{\simeq}{\rightarrow} F_{*} \mathrm{H}^{1} \Omega_{X / k}^{\cdot} \cong F_{*} \mathrm{H}^{1} \mathcal{A}_{X}^{.}
$$

Clearly in degree $0, \mathrm{H}^{0}\left(\psi_{\tilde{X}}\right)=F^{*}=C^{-1}$. Since the vertical arrows in diagram (1.1) are isomorphisms of (sheaves of) graded algebras, it follows that $\mathrm{H}^{*} \mathcal{A}_{X}^{*}$ is a graded-commutative algebra, and therefore

$$
\mathrm{H}^{*}\left(\psi_{\tilde{X}}\right): \bigoplus_{i} \Omega_{X^{\prime} / k}^{i} \rightarrow F_{*} \mathrm{H}^{\cdot} \mathcal{A}_{X}
$$

is a homomorphism of graded algebras. But then $\mathrm{H}^{\cdot}\left(\psi_{\tilde{X}}\right)=C^{-1}$ in all degrees, and it's an isomorphism.

Of course in the derived category the map $\psi_{\tilde{X}}$ is independent of the choices of Frobenius liftings.

\section{A Hodge-Type DeComposition?}

The second aspect is a naive attempt to use adeles for a Hodge-type decomposition of De Rham cohomology. Suppose char $k=0$ and $X$ is smooth over $k$, of dimension $n$. For any $0 \leq p, q \leq n$ define a canonical subspace

$$
\mathrm{H}^{p, q}:=\frac{\Gamma\left(X, \mathcal{A}_{X}^{p, q}\right) \cap \operatorname{Ker} D}{\Gamma\left(X, \mathcal{A}_{X}^{p, q}\right) \cap \operatorname{Im} D} \subset \mathrm{H}_{\mathrm{DR}}^{p+q}(X / k)
$$

(cf. $[\mathrm{GH}]$, p. 116). Since the sheaves $\mathcal{A}_{X}^{p, q}$ imitate the Dolbeault sheaves on a complex manifold so nicely, one can imagine that

$$
\mathrm{H}_{\mathrm{DR}}^{i}(X / k)=\bigoplus_{p+q=i} \mathrm{H}^{p, q}
$$

if $X$ is proper. Yet this is false, as can be seen from the example below.

What we get is a serious breakdown in the analogy to smooth forms on a complex manifold. I should mention that even in [HY2] there was a breakdown in this analogy; there it was not possible to define a connection on the adelic sections of a vector bundle, and hence an auxiliary algebraic device, the sheaf $\tilde{\mathcal{A}}_{X}$ of ThomSullivan adeles, had to be introduced. 
Problem 2.1. Is it true that for $X$ smooth, the filtration on $\mathcal{A}_{X}$ by the subcomplexes $\mathcal{A}_{X}^{\cdot}, \geq q$ induces the coniveau filtration on $\mathrm{H}_{\mathrm{DR}}^{\cdot}(X / k)$ ?

Example 2.2. Suppose $k$ is algebraically closed and $X$ is an elliptic curve. Then $\operatorname{dim} \mathrm{H}_{\mathrm{DR}}^{1}(X / k)=2$. Consider the nondegenerate pairing on $\mathrm{H}_{\mathrm{DR}}^{1}(X / k)$ given by

$$
\langle[\alpha],[\beta]\rangle=\int_{X}[\alpha] \smile[\beta]=\sum_{\xi} \operatorname{Res}_{\xi}(\alpha \cdot \beta)
$$

for adeles $\alpha, \beta \in \mathcal{A}_{X}^{1}$. We see that $\left\langle\mathrm{H}^{1,0}, \mathrm{H}^{1,0}\right\rangle=\left\langle\mathrm{H}^{0,1}, \mathrm{H}^{0,1}\right\rangle=0$. Therefore if $\mathrm{H}_{\mathrm{DR}}^{1}(X)=\mathrm{H}^{1,0}+\mathrm{H}^{0,1}$, then $\operatorname{dim} \mathrm{H}^{1,0}=\operatorname{dim} \mathrm{H}^{0,1}=1$. It is easy to find $0 \neq[\alpha] \in$ $\mathrm{H}^{1,0}$; take any $0 \neq[\alpha] \in \Gamma\left(X, \Omega_{X / k}^{1}\right)$. On the other hand an adele

$$
\beta=\left(b_{(\operatorname{gen}, x)}\right) \in \Gamma\left(X, \mathcal{A}_{X}^{0,1}\right)=\mathbb{A}_{\text {red }}^{1}\left(X, \mathcal{O}_{X}\right)
$$

(where $x$ runs over the set $X_{0}$ of closed points, and gen is the generic point) satisfies $\mathrm{D} \beta=0$ iff $\mathrm{d} b_{(\operatorname{gen}, x)}=0$ for every $x$. This forces $b_{(\operatorname{gen}, x)} \in k$. But taking $b=$ $\left(b_{(\mathrm{gen})}, b_{(x)}\right) \in \mathbb{A}_{\mathrm{red}}^{0}\left(X, \mathcal{O}_{X}\right)$, with $b_{(\mathrm{gen})}=0, b_{(x)}=b_{(\mathrm{gen}, x)}$ we get $\beta=\mathrm{D} b$. Hence $\mathrm{H}^{0,1}=0$.

Problem 2.3. For $\alpha$ as above find explicitly a cocycle $\beta \in \Gamma\left(X, \mathcal{A}_{X}^{1}\right)$ s.t. $\langle\alpha, \beta\rangle=1$.

The best I can do is:

Proposition 2.4. Suppose $X$ is a smooth proper curve and $k$ is algebraically closed. Let $\alpha_{(\mathrm{gen})} \in \Omega_{k(X) / k}^{1}$ be a differential of the 2 -nd kind, namely $\operatorname{Res}_{(\mathrm{gen}, x)} \alpha_{(\mathrm{gen})}=0$ for every $x \in X_{0}$. Then it defines a cocycle $\alpha \in \Gamma\left(X, \mathcal{A}_{X}^{1}\right)$ whose component at (gen) is $\alpha_{(\text {gen) }}$. Every cohomology class in $\mathrm{H}_{\mathrm{DR}}^{1}(X / k)$ is gotten in this way. The Hodge filtration is induced by the differentials of the 1-st kind.

Proof. The adele $\alpha$ will be given by its bihomogeneous components, $\alpha=\alpha^{1,0}+\alpha^{0,1}$. We set $\alpha^{1,0}:=\left(\alpha_{(\operatorname{gen})}, \alpha_{(x)}\right)$ where for $x \in X_{0}, \alpha_{(x)}=0$. Since $\operatorname{Res}_{(\operatorname{gen}, x)} \alpha_{(\operatorname{gen})}=0$ there is some $a_{(\operatorname{gen}, x)} \in k(X)_{(\text {gen }, x)}$ (unique up to adding a constant) s.t. d $a_{(\text {gen }, x)}=$ $\alpha_{(\text {gen })}$. Set $\alpha^{0,1}:=\left(a_{(\operatorname{gen}, x)}\right)$. Then $\alpha$ is evidently a cocycle.

If $\alpha_{(\text {gen })}$ is of the 1-st kind then actually we get $a_{(\text {gen }, x)} \in \mathcal{O}_{X,(x)}$; call this element also $a_{(x)}$. So we can define an adele $\tilde{\alpha}=\tilde{\alpha}^{1,0}+\tilde{\alpha}^{0,1}$ with $\tilde{\alpha}^{1,0}:=\left(\alpha_{(\operatorname{gen})}, \mathrm{d} a_{(x)}\right)$ and $\tilde{\alpha}^{0,1}:=0$. We get a cocycle (cohomologous to $\alpha$ ), and conversely any cocycle in $\Gamma\left(X, \mathcal{A}_{X}^{1,0}\right)$ looks like this.

Consider the niveau spectral sequence of De Rham homology (cf. [Ye3]). A comparison of dimensions shows that this degenerates at the $E_{2}$ term. Also the niveau filtration on $\mathrm{H}_{1}^{\mathrm{DR}}(X / k)$ is trivial. Hence we get

$$
\begin{aligned}
\mathrm{H}_{1}^{\mathrm{DR}}(X / k) & =\operatorname{Ker}\left(\mathrm{H}^{1} \Omega_{k(X) / k} \rightarrow \bigoplus_{x \in X_{0}} k\right) \\
& \cong \text { (forms of the 2-nd kind)/(exact forms). }
\end{aligned}
$$

Now the map $\mathrm{H}_{\mathrm{DR}}^{1}(X / k) \rightarrow \mathrm{H}_{1}^{\mathrm{DR}}(X / k),[\alpha] \mapsto[\alpha] \frown[X]= \pm\left[\mathrm{C}_{X} \cdot \alpha\right]$ is bijective. A direct inspection reveals that the adele $\alpha=\alpha^{1,0}+\alpha^{0,1}$ is sent to the differential of the second kind $\alpha_{(\text {gen })} \in \Omega_{k(X) / k}^{1}$.

\section{REFERENCES}

[Be] A.A. Beilinson, Residues and adeles, Funkt. Anal. Pril. 14 (1980) no. 1, 44-45; English trans. in Func. Anal. Appl. 14 (1980) no. 1, 34-35. MR 81f:14010

[DI] P. Deligne and L. Illusie, Relèvements modulo $p^{2}$ et décomposition du complexe de de Rham, Inv. Math. 89 (1987), 247-270. MR 88j:14029 
[GH] P. Griffiths and J. Harris, "Principles of Algebraic Geometry", Wiley, New York, 1978. MR 80b:14001

[Hr] A. Huber, On the Parshin-Beilinson Adeles for Schemes, Abh. Math. Sem. Univ. Hamburg 61 (1991), 249-273. MR 92k:14024

[HY1] R. Hübl and A. Yekutieli, Adeles and differential forms, to appear: J. reine angew. Math.

[HY2] R. Hübl and A. Yekutieli, Adelic Chern forms and the Bott residue formula, preprint (1994).

[Ye1] A. Yekutieli, "An Explicit Construction of the Grothendieck Residue Complex" (with an appendix by P. Sastry), Astérisque 208 (1992). MR 94e: 14026

[Ye2] A. Yekutieli, Smooth formal embeddings, preprint (1995).

[Ye3] A. Yekutieli, Residues and differential operators on schemes, preprint (1994).

Department of Theoretical Mathematics, The Weizmann Institute of Science, ReHOVot 76100, ISREAL

E-mail address: amnon@wisdom.weizmann.ac.il 